\title{
Environmental microbial contaminants in selected retail pharmacies of Sri Lanka
}

\author{
A Thayananthan, TMIUK Tennakoon
}

\section{Introduction}

Maintaining the microbial quality of the environment is crucial in pharmaceutical institutions, especially community pharmacies, since it has a direct relationship with patients. The sterility of environmental surfaces of pharmacies can be maintained by proper cleaning and with disinfectants. Microbial contamination is the primary reason for product spoilage or deterioration. The present study was carried out to assess the sanitation practice of pharmacies on maintenance of cleanliness in the premises by isolation, identification and enumerating the bacterial concentration from samples collected from selected pharmacies.

\section{Methods}

Environmental samples were collected from four places (storeroom, extemporaneous preparation room, equipment, and hands of personnel) in five selected retail pharmacies. Swab samples were taken using sterile cotton swabs after wetting with normal saline and air samples collected using the settle plate method. The collected swab samples were sent to the laboratory in an airtight container under aseptic conditions. The samples were plated on blood agar plates. Air samples were collected by placing two blood agar plates under the unidirectional airflow inside the storeroom. For the colony forming units (CFU) counts, the samples were incubated for 24 hours while surface samples were incubated up to 72 hours. After the purification of colonies, standard bacterial identification tests were performed.

\section{Results}

In total, 33 bacterial species were identified from the pharmacies. Of these, 31 were Gram positive (29 coagulase negative staphylococci (CNS) and two Streptococcus spp. while 2 were Gram negative (Pseudomonas aeruginosa and Proteus vulgaris). The CFU count of air samples after one-hour exposure under Uni-directional air flow was less than 20 CFU except from one pharmacy which was 59.

\section{Conclusions}

Pharmacies under investigation were confirmed to have poor sterility control practices as they have shown the growth of both Gram negative bacilli ( $P$. aeruginosa) and Gram positive cocci (CNS), of which the latter was in more abundance. These findings do not comply with the standard of United State Pharmacopoeia guidelines.

Keywords: Sterility, Environmental monitoring, Extemporaneous preparation, Disinfectant

Department of Pharmacy, Faculty of Allied Health Sciences, University of Peradeniya, Sri Lanka 